\title{
Study of Nuts and Dried Fruits Consumption in Adolescents in Relation to Risk of Metabolic Syndrome and Its Components: Tehran Lipid and Glucose Study
}

\author{
Parvin Mirmiran ${ }^{1}$, Shirin Ghotboddin Mohammadi ${ }^{1}$, Zahra Bahadoran ${ }^{1}$, Fereidoun Azizi ${ }^{2, ~ * ~}$ \\ ${ }^{1}$ Nutrition and Endocrine Research Center, Research Institute for Endocrine Sciences, Shahid Beheshti University of Medical Sciences, \\ Tehran, Iran \\ ${ }^{2}$ Endocrine Research Center, Research Institute of Endocrinology and Metabolism, Shahid Beheshti University of Medical Sciences, Tehran, \\ Iran
}

Email address:

azizi@endocrine.ac.ir (F. Azizi)

To cite this article:

Parvin Mirmiran, Shirin Ghotboddin Mohammadi, Zahra Bahadoran, Fereidoun Azizi. Study of Nuts and Dried Fruits Consumption in Adolescents in Relation to Risk of Metabolic Syndrome and Its Components: Tehran Lipid and Glucose Study. International Journal of Nutrition and Food Sciences. Special Issue: Nutrition and Cardiometabolic Risk Factors. Vol. 5, No. 1-2, 2016, pp. 8-13. doi: $10.11648 /$ j.ijnfs.s.2016050102.12

\begin{abstract}
Background and aim. This study was conducted to investigate the association of consumption of nuts and dried fruits with the risk of metabolic syndrome (MetS) and its components adolescents. Material and Methods. In this crosssectional study was conducted in 785 adolescents, aged 10-19 y, participated in the fourth phase of the Tehran Lipid and Glucose Study. Demographics, anthropometrics and biochemical measurements were evaluated. MetS and its components were defined based on a modified definition of de Ferranti. Usual dietary intakes were assessed using a validated semiquantitative food frequency questionnaire. Dried fruits and nuts were included dried figs, raisins, dried berries, peanuts, almonds, walnuts, pistachios, hazelnuts and seeds. To determine the prevalence of metabolic syndrome in quartiles of dried fruits and nuts consumption, logistic regression models adjusted for confounding variables were used. Results. Mean age and body mass index of participants (47\% male) was $14.8 \pm 2.9$ years, and $22.1 \pm 4.7 \mathrm{~kg} / \mathrm{m} 2$, respectively. The prevalence of MetS was $22.2 \%$ (girls: $19.5 \%$ and boys: $25.2 \%$ ). In the highest compared to lowest quartile of nuts and dried fruits, the prevalence of lipid disorders, abdominal obesity and MetS was non-significantly lower. Serum triglycerides significantly decreased across increasing consumption of nuts and dried fruits $(\mathrm{P}<0.05)$. After adjustment for confounding variables, the odds of MetS in the highest compared to the lowest quartile of nuts and dried fruits were 0.65 (95\% CI: 0.4-1.1). Conclusion. Increased consumption of nuts and dried fruits could have favorable effect in prevention of metabolic syndrome and its components.
\end{abstract}

Keywords: Metabolic Syndrome, Dried Fruits, Nuts, Children and Adolescent

\section{Introduction}

Metabolic syndrome (MetS), a risk factor for development of type 2 diabetes and cardiovascular disease, is a cluster of metabolic abnormalities including abdominal obesity, impaired glucose homeostasis, dyslipidemia and hypertension [1]. Studies show that the prevalence of MetS is on the rise with progressive increase in the prevalence of overweight and obesity. Among the risk factors of metabolic syndrome, diet is an important modifiable risk factor [2].

Nuts and dried fruits are rich sources of dietary fiber, various phytochemicals and essential minerals; studies showed that regular consumption of nuts and dried fruits may increase satiety and reduce energy intake and decrease the risk of obesity and type 2 diabetes [3]. Nuts as an important component of the Mediterranean diet could improve insulin sensitivity, and reduce the risk of MetS, cardiovascular disease and diabetes [4]. However considering the inconsistencies in current data, we assessed the association of dried fruits and nuts with the risk of MetS and its components in adolescent participants of the Tehran Lipid and Glucose Study.

\section{Material and Methods}

Subjects

The current study, conducted within the framework of the 
Tehran Lipid and Glucose Study (TLGS), is a prospective study aiming to determine the prevalence of risk factors for non-communicable diseases and improving lifestyles in order to prevent or eliminate risk factors in district 13 of Tehran. The first phase of TLGS was cross-sectional, in which 15005 residents of district 13 of Tehran were selected by a multistage cluster, random sampling method between 19992001. In the fourth phase of TLGS (2008-2011), 935 adolescents, aged 10 to 19 years with complete data were enrolled [5]. Participants with incomplete anthropometric and biochemical measurement data $(\mathrm{n}=8)$, individuals taking drugs that affect the metabolism of glucose, lipids and blood pressure, such as steroids, non-steroidal antiinflammatory drugs, male and female hormones and thyroid hormones $(\mathrm{n}=17)$, individuals with a history of heart disease $(\mathrm{n}=17)$, or those on special diets $(\mathrm{n}=21)$, boys with energy intake $<800$ or $>5000 \mathrm{kcal} / \mathrm{d}$, and the girls with energy intake $<600$ or $>4500 \mathrm{kcal} / \mathrm{d}(\mathrm{n}=87)$ were excluded from the analyses [6]. Final analysis was conducted on the data of 785 participants.

\section{Anthropometric measurements}

Weight was measured with using digital scales (model 707, range $0.1-150 \mathrm{~kg}$, Seca, Hamburg, Germany), with minimally participants clothed and without shoes, to the nearest $100 \mathrm{~g}$. Height was measured with a tape measure (model 208 Portable Body Meter Measuring Device; Seca), while the subjects were in a standing position with shoulders in a normal state and not wearing shoes. Waist circumference (WC), parallel to the belly button, was assessed at the end of a normal expiration. WC was measured with the use of an unstretched tape measure, without any pressure to body surface and recorded to the nearest $1 \mathrm{~cm}$, while the person was wearing cloth that did not change in waist size [5]. BMI was calculated by dividing weight $(\mathrm{kg})$ by the square of height $\left(\mathrm{m}^{2}\right)$. Those with BMI $<85$ th percentile for the age and sex of the Iranian population (based on the CASPIAN study) were considered normal while subjects with a $\mathrm{BMI} \geq 85$ th percentile and $<95$ th percentile at risk for overweight and those with $\mathrm{BMI} \geq 95$ th percentile were considered overweight [7].

\section{Assessment of dietary intake}

Usual dietary intake was assessed with the use of a 147item food frequency questionnaire (FFQ). The FFQ consisted of a list of foods with standard serving sizes. Participants were asked to report their frequency of consumption of each food item during the previous year on a daily, weekly, or monthly basis. The reliability of the FFQ was evaluated in a randomly chosen subgroup of 132 subjects by comparing nutrient consumption ascertained by FFQ responses on 2 occasions. The correlation coefficients for the repeatability of cheese, milk, and yoghurt were 0.73, 0.69, and 0.79, respectively. Comparative validity was determined by comparison with intakes estimated from the average of twelve 24-h dietary recalls (one for each month of the year). Overall, these data indicate that the FFQ provides reasonably valid measures of the average long term dietary intake [8]. Portion sizes of consumed foods were converted from household measures to grams [9]. Considering that the Iranian food composition table is incomplete [10] in the number of food items and micronutrients, for the analysis of energy and nutrient intake of most food items, except for items like kashk, the USDA food composition table was used [11]. In this study, nuts and dried fruits was defined as sum of dried figs, raisins, dried berries, peanuts, almonds, walnuts, pistachios, hazelnuts and seeds.

\section{Blood pressure measurement}

Using a mercury sphygmomanometer and the Korotkoff sound technique, after resting for at least 5 minutes sitting on a chair, blood pressure was measured twice on the right arm, with a minimum interval of 30 seconds and an accuracy of 2 $\mathrm{mm} \mathrm{Hg}$; the average of two measurements was considered as the final pressure. Systolic blood pressure with the first sound to be heard and diastolic blood pressure with the disappearance of sound were recorded [5].

\section{Biochemical measurements}

All tests were done at the Tehran lipid and glucose research center lab on the same day as sampling. From all subjects after 12-14 hours of fasting during the night, between 7-9 am, venous blood samples were obtained to measure blood glucose level and lipid levels including triglycerides and HDL-C; samples were centrifuged within 30-45 minutes of collection. Blood glucose was measured on the day of blood collection using the enzymatic colorimetric method with glucose oxidase. Serum concentration of triglycerides was measured using commercially available enzymatic reagents (Pars Azmoon, Tehran, Iran) adapted to the Selectra auto analyzer. Inter- and intra-assay CVs were $1.6 \%$ and $0.6 \%$ respectively for triglycerides. HDL-C was measured after precipitation of the apolipoprotein $\mathrm{B}$ with a solution of phosphotungstic acid. Inter- and intra-assay CVs were $2 \%$ and $0.5 \%$ for HDL-C, respectively [5].

Definition of metabolic syndrome and its components

In this study MetS in adolescents was defined, based on a modified definition of de Ferranti [12], as having at least 3 of the following components: Fasting plasma glucose concentration $\geq 110 \mathrm{mg} / \mathrm{dL}$, fasting serum triglycerides $\geq 100$ $\mathrm{mg} / \mathrm{dL}, \mathrm{HDL}-\mathrm{C}<45 \mathrm{mg} / \mathrm{dL}$ for boys, aged 15-19 years and $<50 \mathrm{mg} / \mathrm{dL}$ for others; $\mathrm{WC} \geq 75$ th percentile for the age and sex of Iranian population (based on the CASPIAN study) [13]; systolic and diastolic blood pressure $>$ 90th percentile for age, sex, and height based on the recommendations of National Heart, Lung and Blood Institute. Since WC percentiles for Iranian populations have been reported only up to the age of 18 years for individuals aged $>18$, high WC was considered based on the criteria of Iranian adults ( $>$ $95 \mathrm{~cm}$ in both sexes) [14]. To assess systolic and diastolic blood pressure, on the basis of height-for-age charts of CDC [15], height percentile was determined for each person for ages 1 to 17 years using tables published by the National High Blood Pressure Education Program (NHBPEP) according to sex, age and height percentile 90th percentile of systolic and diastolic blood pressure were obtained [16]. To investigate hypertension in persons over 17 years, NCEP criteria for adults were used [17]. 


\section{Statistical analysis}

We used SPSS software (version 16; SPSS Inc, Chicago IL) for all statistical analyses. Normal distribution of the data was evaluated by diagrams and the Kolmogorov-Smirnov test. All components of MetS except triglycerides had normal distribution. To comparing quantitative variables between subjects with and without MetS and also between genders, independent $\mathrm{t}$-test, to compare qualitative variables ratio, the Chi-square test, and to adjust for confounding variables, covariance analysis (ANCOVA) were used. To calculate the odds ratio $(95 \%$ confidence interval) for the MetS and its components in quartiles of nuts and dried fruit, logistic regression models with adjustment for age, sex, body mass index and energy intake were used.

\section{Results}

Mean \pm SD for age of participants (52.9\% girls) was 14.8 \pm 2.9 years. Mean \pm SD for BMI was $22.1 \pm 4.7 \mathrm{~kg} / \mathrm{m}^{2}$. Demographic characteristics and components of MetS, in adolescent participants, both healthy subjects and those with MetS, are shown in Table 1.

Table 1. Demographic Characteristics and The Components of MetS in Healthy Subjects and Subjects with MetS.

\begin{tabular}{|c|c|c|c|}
\hline & Healthy subjects & Subjects with MetS & P \\
\hline & $(n=611)$ & $(n=174)$ & $P$ \\
\hline $\operatorname{BMI}\left(\mathrm{kg} / \mathrm{m}^{2}\right)$ & $21.1 \pm 4.2$ & $25.2 \pm 4.4$ & 0.005 \\
\hline Fasting blood glucose (mg/dL) & $91.8 \pm 8.1$ & $94.6 \pm 7.8$ & $<0.001$ \\
\hline Triglycerides $(\mathrm{mg} / \mathrm{dL})$ & $74(60-94)$ & $131(113-166)$ & $<0.001$ \\
\hline HDL cholesterol (mg/dL) & $52.5 \pm 10.3$ & $40.9 \pm 6.9$ & $<0.001$ \\
\hline Waist circumferences $(\mathrm{cm})$ & $100 \pm 11.6$ & $86.3 \pm 10.9$ & $<0.001$ \\
\hline Systolic blood pressure (mmHg) & $66.2 \pm 9.4$ & $107 \pm 13.0$ & $<0.001$ \\
\hline Diastolic blood pressure $(\mathrm{mmHg})$ & & $70.3 \pm 11.6$ & $<0.001$ \\
\hline
\end{tabular}

Data are mean $\pm \mathrm{SD}$ except for TG. For TG median and interquartile ranges are reported. P value except for TG were obtained by using t-test and for TG was obtained by using Mann-Whitney U test.

The prevalence of MetS and its components across quartiles of nuts and dried fruits consumption are presented in Table 2. Across increasing consumption of nuts and dried fruits, the prevalence of abdominal obesity and metabolic syndrome nonsignificantly decreased.

Table 2. Prevalence of MetS and Its Components across Quartiles of Nuts and Dried Fruits Intake.

\begin{tabular}{|c|c|c|c|c|c|}
\hline \multicolumn{6}{|c|}{ Quartiles of nuts and dried fruits } \\
\hline & Q1 & Q2 & Q3 & Q4 & $\mathbf{P}^{1}$ \\
\hline High blood glucose (\%) & 1.0 & 2.1 & 1.0 & 1.0 & 0.74 \\
\hline High triglycerides (\%) & 37.4 & 40.5 & 32.0 & 29.7 & 0.10 \\
\hline Low HDL-C (\%) & 56.1 & 48.2 & 49.7 & 49.2 & 0.39 \\
\hline Abdominal obesity (\%) & 55.1 & 53.3 & 46.2 & 47.7 & 0.23 \\
\hline Elevated blood pressure (\%) & 12.1 & 12.8 & 6.6 & 12.8 & 0.14 \\
\hline Metabolic syndrome (\%) & 25.3 & 25.1 & 19.3 & 19.0 & 0.24 \\
\hline
\end{tabular}

Lowest to highest quartiles of total nuts and dried fruits intakes were: $<5.0,5.0-8.7,8.7-15.5,>15.5 \mathrm{~g} / \mathrm{d}$ respectively

${ }^{1}$ Chi square test was used to compare the prevalence of MetS and its components across quartile categories of nuts and dried fruits consumption.

Adjusted mean components of MetS according to quartiles of nuts and dried fruits intake are shown in Table 3. After adjustment for age, sex, energy intake, no significant differences in the components of MetS in quartiles of nuts and dried fruits were observed; only the concentration of triglycerides significantly decreased in the higher compared the lower quartile of nuts and dried fruits intakes. Also with increased intake of nuts and dried fruits, a non-significant decreasing trend was observed in waist circumference, systolic and diastolic blood pressure.

Table 3. Adjusted means for components of MetS across quartiles of nuts and dried fruits intake.

\begin{tabular}{|c|c|c|c|c|c|}
\hline \multicolumn{6}{|c|}{ Quartiles of nuts and dried fruits intake } \\
\hline & $Q 1$ & $Q 2$ & Q3 & $Q 4$ & $P^{l}$ \\
\hline Fasting blood glucose (mg/dL) & $92.9 \pm 0.6$ & $92.4 \pm 0.6$ & $91.9 \pm 0.6$ & $92.5 \pm 0.6$ & 0.70 \\
\hline Triglycerides (mg/dL) & $83(68-118)$ & $93(67-118)$ & $84(62.5-112)$ & $78(60-108)$ & 0.04 \\
\hline HDL cholesterol (mg/dL) & $49.5 \pm 0.8$ & $50.1 \pm 0.8$ & $50.2 \pm 0.8$ & $49.9 \pm 0.8$ & 0.95 \\
\hline Waist circumference $(\mathrm{cm})$ & $78.2 \pm 0.8$ & $77.0 \pm 0.8$ & $76.3 \pm 0.8$ & $75.9 \pm 0.8$ & 0.24 \\
\hline Systolic blood pressure (mmHg) & $102 \pm 0.9$ & $102 \pm 0.8$ & $101 \pm 0.9$ & $101 \pm 0.8$ & 0.24 \\
\hline
\end{tabular}

Data are mean \pm SD except for TG. For TG median and inter-quartile range is reported.

${ }^{1}$ Analysis of covariance with adjustment for age, sex and energy intake was used. P value for TG was obtained using Kruskal Wallis test. 
The odds $(95 \% \mathrm{CI})$ of MetS and its components across quartile categories of nuts and dried fruits intake are shown in Table 4. After adjustment for age, sex, body mass index and energy intake, no significant differences in the risk of MetS and its components was observed in the quartile categories of nuts and dried fruits. Across increasing consumption of nuts and dried fruits, the odds of MetS and abdominal obesity non-significantly decreased.

Table 4. Multivariate-Adjusted Odds Ratios and 95\% Confidence Interval for MetS and Its Components across Quartiles of Nuts and Dried Fruits Intake.

\begin{tabular}{|c|c|c|c|c|c|}
\hline \multicolumn{6}{|c|}{ Quartiles of nuts and dried fruits intake } \\
\hline & $Q 1$ & $Q 2$ & $Q 3$ & Q4 & $P^{l}$ \\
\hline Metabolic syndrome & 1.00 & $0.89(0.5-1.5)$ & $0.63(0.4-1.1)$ & $0.65(0.4-1.1)$ & 0.19 \\
\hline Abdominal obesity & 1.00 & $0.93(0.6-1.4)$ & $0.70(0.4-1.1)$ & $0.74(0.5-1.1)$ & 0.25 \\
\hline Low HDL-C & 1.00 & $0.82(0.5-1.2)$ & $0.88(0.6-1.3)$ & $0.80(0.5-1.2)$ & 0.73 \\
\hline High triglycerides & 1.00 & $1.1(0.7-1.7)$ & $0.77(0.5-1.2)$ & $0.70(0.5-1.1)$ & 0.12 \\
\hline High blood glucose & 1.00 & $0.98(0.2-6.4)$ & $0.40(0.04-3.6)$ & $0.61(0.1-4.9)$ & 0.75 \\
\hline Elevated blood pressure & 1.00 & $1.1(0.6-2.2)$ & $0.53(0.2-1.1)$ & $1.1(0.58-2.0)$ & 0.17 \\
\hline
\end{tabular}

Lowest to highest quartiles of total nuts and dried fruits intake respectively were: $<5.0,5.0-8.7,8.7-15.5,>15.5 \mathrm{~g} / \mathrm{d}$.

Logistic regression models with adjustment for age, sex, and energy intake were used.

\section{Discussion}

The present study investigated the relationship between nuts and dried fruits consumption and MetS and its components in 10-19 year old adolescent participants of the fourth phase of the TLGS. The prevalence of MetS was 22.2\% (girls: $19.5 \%$ and boys: $25.2 \%$ ). The results showed that higher consumption of nuts and dried fruits could have an inverse association with metabolic disorders especially dyslipidemia and abdominal obesity.

Similarly, in a cross sectional study no significant association was observed between consumption of nuts and hyperglycemia, hypertension, serum triglycerides and HDLC levels [4]. Several prospective cohort studies show that consumption of dried fruits and nuts are inversely associated with BMI and risk of obesity [18-21]. Between the years 1999-2004, in the NHANES cohort, nuts consumption associated with a lower incidence of risk factors for cardiovascular disease and the MetS [2]. The SUN cohort study with a 6 month follow-up, reported that compared to those who did not use any nuts, those who consumed $>2$ servings of nuts per week had a 32\% lower risk of MetS [22]. In the Physicians' Health Study, a lower incidence of hypertension was observed in people who regularly consumed nuts; however, this association was observed mainly among lean subjects $(\mathrm{BMI}<25)$ while in overweight and obese subjects there was no correlation [23]; Contrary to their study, the SUN cohort study found no association between consumption of nuts with hypertension [24]. In the NHANES study, the risk of low HDL-C in consumers of nuts and peanuts was $20 \%$ lower than others [25]. Pooled analysis of the recent clinical studies shows that consumption of nuts has no effect on HDL-C [26].

Nuts are high energy density foods, and approximately $45-$ $75 \%$ of the weight is composed of fat [27]. The fatty acid composition of nuts is beneficial because the saturated fatty acid content is low (16-4\%) and because they are rich in mono-unsaturated fatty acids (oleic acid) and polyunsaturated fatty acids (linoleic acid) [4, 27]. Nuts are a good source of protein (approximately $25 \%$ of energy) and often have high content of $\mathrm{L}$ - arginine, an amino acid which is the precursor of the endogenous vasodilator, nitric oxide [27]. Nuts are also rich sources of fiber, which ranges from 4 to 11 grams of fiber per 100 grams of nuts [27, 28]. Nuts contain a variety of vitamins (such as folic acid, niacin, vitamin $\mathrm{E}$ tocopherols and $\mathrm{B}_{6}$ ) and minerals (such as calcium, magnesium, potassium, selenium, and copper) [27, 29].

As mentioned nuts contain minerals such as calcium, magnesium, and potassium and the sodium content of raw or roasted but otherwise unprocessed nuts is very low [30]. High intakes of calcium, magnesium, and potassium, together with a low sodium intake, are associated with protection against arterial hypertension, bone demineralization, insulin resistance, and overall cardiovascular risk [31].

Nuts contain sizeable amount of folate [30], a B-vitamin necessary for normal cellular function that plays an important role in detoxification homocysteine, a sulfur containing amino acid with atherothrombotic properties that accumulates in plasma when folate status is subnormal [32].

Also nuts contain several bioactive compounds, including phytosterols and phenolic compounds, especially Flavonoids and proanthocyanidins [27, 29].

The mechanism of the action of phytosterols has been linked to their hydrophobicity, which is higher than that of cholesterol because of a bulkier hydrocarbon molecule and entails a higher affinity for micelles than cholesterol. Therefore the phytosterols content of nuts contributes to their cholesterol-lowering effect [33].

The high content of phenolic compounds in nuts, particularly in the pellicle, might anticipate an antiinflammatory effect of nut consumption [34]. Walnuts have more anti-inflammatory effects than other nuts for two reasons; Firstly, because they are the only nuts that contain substantial amounts of ALA, which is known as one of the more anti-inflammatory fatty acids $[35,36]$, and secondly, they are also particularly rich in the phenolic ellagic acid, which has shown potent anti-inflammatory properties in experimental studies [37, 38].

Regarding study strengths and limitation, one limitation of this study is its cross-sectional design, which precludes inferences on causation relationship between the parameters. 
Also due to the incomplete Iranian food composition table in the number of food items and micronutrients, for most food items, except for items like kashk the USDA food composition table was used. Although the effect of confounders were considered in this study as much as possible, but we were unable to control for the effect of genetic factors on MetS. The main strength of this study was population-based design.

In conclusion, nuts and dried fruits are nutrient rich foods with wide ranging cardiovascular and metabolic benefits, and they can readily be incorporated into healthy diets, demonstrating that nuts and dried fruits in the diet improve nutritional quality [28].

\section{Acknowledgment}

We thank the TLGS participants and the field investigators of the TLGS for their assistance in physical examinations, biochemical and nutritional evaluation and database management. This study was supported by grant 121 from National Research Council of the Islamic Republic of Iran and the Research Institute for Endocrine Sciences of Shahid Beheshti University of Medical Sciences. The authors acknowledge Ms. Niloofar Shiva for critical editing of English grammar and syntax of the manuscript.

\section{References}

[1] Lutsey PL, Steffen LM, Stevens J. Dietary intake and the development of the metabolic syndrome: the Atherosclerosis Risk in Communities study. Circulation. 2008 Feb 12; 117(6): 754-61.

[2] O'Neil CE, Keast DR, Nicklas TA, Fulgoni VL, 3rd. Nut consumption is associated with decreased health risk factors for cardiovascular disease and metabolic syndrome in U.S. adults: NHANES 1999-2004. J Am Coll Nutr. 2011 Dec; 30(6): 502-10.

[3] Brennan AM, Sweeney LL, Liu X, Mantzoros CS. Walnut consumption increases satiation but has no effect on insulin resistance or the metabolic profile over a 4-day period. Obesity (Silver Spring). 2010 Jun; 18(6): 1176-82.

[4] Ibarrola-Jurado N, Bullo M, Guasch-Ferre M, Ros E, Martinez-Gonzalez MA, Corella D, et al. Cross-sectional assessment of nut consumption and obesity, metabolic syndrome and other cardiometabolic risk factors: the PREDIMED study. PLoS One. 2013; 8(2): e57367.

[5] Azizi F, Ghanbarian A, Momenan AA, Hadaegh F, Mirmiran P, Hedayati M, et al. Prevention of non-communicable disease in a population in nutrition transition: Tehran Lipid and Glucose Study phase II. Trials. 2009; 10(1): 5.

[6] Willett W. Issues in analysis and presentation of dietary data. Nutritional epidemiology. 1998; 2: 321-45.

[7] Kelishadi R, Ardalan G, Gheiratmand R, Majdzadeh R, Hosseini M, Gouya M, et al. Thinness, overweight and obesity in a national sample of Iranian children and adolescents: CASPIAN Study. Child: care, health and development. 2008; 34(1): 44-54.
[8] Mirmiran P, Hosseini Esfahani F, Mehrabi Y, Hedayati M, Azizi F. Reliability and relative validity of an FFQ for nutrients in the Tehran Lipid and Glucose Study. Public health nutrition. 2010; 13(05): 654-62.

[9] Ghaffarpour M, Houshiar-Rad A, Kianfar H. The manual for household measures, cooking yields factors and edible portion of foods. Tehran: Nashre Olume Keshavarzy. 1999: 1-40.

[10] Azar M, Sarkisian E. Food composition table of Iran. Tehran: National Nutrition and Food Research Institute, Shaheed Beheshti University. 1980; 65.

[11] USDA. USDA national nutrient database for standard reference, release 21. US Department of Agriculture; 2011 [cited 2011]; Available from: http://www.nal.usda.gov/fnic/foodcomp/search/.

[12] de Ferranti SD, Gauvreau K, Ludwig DS, Neufeld EJ, Newburger JW, Rifai N. Prevalence of the metabolic syndrome in American adolescents findings from the Third National Health and Nutrition Examination Survey. Circulation. 2004; 110(16): 2494-7.

[13] Kelishadi R, Gouya MM, Ardalan G, Hosseini M, Motaghian M, Delavari A, et al. First reference curves of waist and hip circumferences in an Asian population of youths: CASPIAN study. Journal of tropical pediatrics. 2007; 53(3): 158-64.

[14] Fereidoun Azizi M, Davood Khalili M, Hassan Aghajani M, Alireza Esteghamati M, Farhad Hosseinpanah M, Alireza Delavari M, et al. Appropriate waist circumference cut-off points among Iranian adults: the first report of the Iranian National Committee of Obesity. Archives of Iranian medicine. 2010; 13(3): 243.

[15] CDC. Data Table of Stature-for-age Charts. 2012 [cited 2012 23 jun]; Available from: http://www.cdc.gov/growthcharts/htmlcharts/statge.htm.

[16] NHBPEP. The fourth report on the diagnosis, evaluation, and treatment of high blood pressure in children and adolescents. Pediatrics. 2004; 114(2): 555-76.

[17] Grundy SM, Cleeman JI, Daniels SR, Donato KA, Eckel RH, Franklin BA, et al. Diagnosis and management of the metabolic syndrome an American Heart Association/National Heart, Lung, and Blood Institute scientific statement. Circulation. 2005; 112(17): 2735-52.

[18] Bes-Rastrollo M, Sabate J, Gomez-Gracia E, Alonso A, Martinez JA, Martinez-Gonzalez MA. Nut consumption and weight gain in a Mediterranean cohort: The SUN study. Obesity (Silver Spring). 2007 Jan; 15(1): 107-16.

[19] Casas-Agustench P, Lopez-Uriarte P, Bullo M, Ros E, CabreVila JJ, Salas-Salvado J. Effects of one serving of mixed nuts on serum lipids, insulin resistance and inflammatory markers in patients with the metabolic syndrome. Nutr Metab Cardiovasc Dis. 2011 Feb; 21(2): 126-35.

[20] Bes-Rastrollo M, Wedick NM, Martinez-Gonzalez MA, Li TY, Sampson L, Hu FB. Prospective study of nut consumption, long-term weight change, and obesity risk in women. Am J Clin Nutr. 2009 Jun; 89(6): 1913-9.

[21] Martinez-Gonzalez MA, Bes-Rastrollo M. Nut consumption, weight gain and obesity: Epidemiological evidence. Nutr Metab Cardiovasc Dis. 2011 Jun; 21 Suppl1: S40-5. 
[22] Fernandez-Montero A, Bes-Rastrollo M, Beunza JJ, BarrioLopez MT, de la Fuente-Arrillaga C, Moreno-Galarraga L, et al. Nut consumption and incidence of metabolic syndrome after 6-year follow-up: the SUN (Seguimiento Universidad de Navarra, University of Navarra Follow-up) cohort. Public Health Nutr. 2013 Nov; 16(11): 2064-72.

[23] Djousse L, Rudich T, Gaziano JM. Nut consumption and risk of hypertension in US male physicians. Clin Nutr. 2009 Feb; 28(1): 10-4.

[24] Martinez-Lapiscina EH, Pimenta AM, Beunza JJ, BesRastrollo M, Martinez JA, Martinez-Gonzalez MA. Nut consumption and incidence of hypertension: the SUN prospective cohort. Nutr Metab Cardiovasc Dis. 2010 Jun; 20(5): 359-65.

[25] O'Neil CE, Keast DR, Nicklas TA, Fulgoni VL, 3rd. Out-ofhand nut consumption is associated with improved nutrient intake and health risk markers in US children and adults: National Health and Nutrition Examination Survey 1999-2004. Nutr Res. 2012 Mar;32(3):185-94.

[26] Sabate J, Oda K, Ros E. Nut consumption and blood lipid levels: a pooled analysis of 25 intervention trials. Arch Intern Med. 2010 May 10; 170(9): 821-7.

[27] Ros E. Health benefits of nut consumption. Nutrients. 2010 Jul; 2(7): 652-82.

[28] Kendall CW, Josse AR, Esfahani A, Jenkins DJ. Nuts, metabolic syndrome and diabetes. Br J Nutr. 2010 Aug; 104(4): 465-73.

[29] Coates AM, Howe PR. Edible nuts and metabolic health. Curr Opin Lipidol. 2007 Feb; 18(1): 25-30.

[30] Segura R, Javierre C, Lizarraga MA, Ros E. Other relevant components of nuts: phytosterols, folate and minerals. $\mathrm{Br} \mathrm{J}$ Nutr. 2006 Nov; 96 Suppl 2: S36-44.
[31] Cordain L, Eaton SB, Sebastian A, Mann N, Lindeberg S, Watkins BA, et al. Origins and evolution of the Western diet: health implications for the 21 st century. Am J Clin Nutr. 2005 Feb; 81(2): 341-54.

[32] Nath KA. Homocysteine and atherothrombosis. N Engl J Med. 1998 Aug 13; 339(7): 478-9.

[33] Garrido I, Monagas M, Gomez-Cordoves C, Bartolome B. Polyphenols and antioxidant properties of almond skins: influence of industrial processing. J Food Sci. 2008 Mar; 73(2): C106-15.

[34] Rahman I, Biswas SK, Kirkham PA. Regulation of inflammation and redox signaling by dietary polyphenols. Biochem Pharmacol. 2006 Nov 30; 72(11): 1439-52.

[35] Zhao G, Etherton TD, Martin KR, Gillies PJ, West SG, KrisEtherton PM. Dietary alpha-linolenic acid inhibits proinflammatory cytokine production by peripheral blood mononuclear cells in hypercholesterolemic subjects. Am J Clin Nutr. 2007 Feb; 85(2): 385-91.

[36] Zhao G, Etherton TD, Martin KR, West SG, Gillies PJ, KrisEtherton PM. Dietary alpha-linolenic acid reduces inflammatory and lipid cardiovascular risk factors in hypercholesterolemic men and women. J Nutr. 2004 Nov; 134(11): 2991-7.

[37] Papoutsi Z, Kassi E, Chinou I, Halabalaki M, Skaltsounis LA, Moutsatsou P. Walnut extract (Juglans regia L.) and its component ellagic acid exhibit anti-inflammatory activity in human aorta endothelial cells and osteoblastic activity in the cell line KS483. Br J Nutr. 2008 Apr; 99(4): 715-22.

[38] Karlsson S, Nanberg E, Fjaeraa C, Wijkander J. Ellagic acid inhibits lipopolysaccharide-induced expression of enzymes involved in the synthesis of prostaglandin E2 in human monocytes. Br J Nutr. 2010 Apr; 103(8): 1102-9. 